\title{
Rust effector PNPi interacting with wheat TaPR1a attenuates plant defense response
}

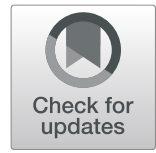

\author{
Weishuai Bi', Shuqing Zhao', Jiaojie Zhao', Jun Su', Xiumei Yu², Daqun Liu', Zhensheng Kang ${ }^{3}$, \\ Xiaojie Wang ${ }^{3^{*}}$ and Xiaodong Wang ${ }^{1 *}$ (iD
}

\begin{abstract}
NPR1 is a key regulator of systemic acquired resistance (SAR) in plant species. In our previous study, we identified a conserved fungal effector PNPi from Puccinia striiformis f. sp. tritici (Pst) that can suppress acquired resistance in local leaf by directly targeting the wheat NPR1 protein. In this investigation, we identified and validated a novel protein interaction between PNPi and wheat pathogenesis-related TaPR1a in the apoplastic space. TaPR1a-overexpressing wheat lines exhibited enhanced resistance to both Pst and Puccinia triticina (Pt). We further determined that exogenous expression of PNPi RNA in transgenic wheat lines reduced the degree of acquired resistance to Magnaporthe oryzae isolate P131 in the region adjacent to Pseudomonas syringae pv. tomato DC3000 infection area. Additionally, when PNPi was overexpressed, the expression levels of two plant defense responsive genes were suppressed upon $P$. syringae DC3000 infection in the local infiltration region. These findings established the mechanism of a single rust effector that can suppress multiple defense responses in wheat plants by targeting different components.
\end{abstract}

Keywords: Wheat rust, Effector, Apoplastic space, Pathogenesis-related protein, Transcriptional response

\section{Background}

Plants are constantly subjected to biotic and abiotic stresses, triggering various plant resistance mechanisms that are mediated by different hormones, such as salicylic acid (SA), jasmonate (JA), and abscisic acid (ABA) (Smith et al. 2017). $\mathrm{SA}$ is a crucial component of plant disease resistance, mediating plant immune response to pathogen infection and generating systemic acquired resistance (SAR) beyond the infection area (Ryals et al. 1995). NPR1 (Nonexpresser of $\underline{P R}$ genes 1 , also known as NIM1 and SAI1) is a key transcriptional regulator of SAR in Arabidopsis. Upon pathogen infection or SA treatment, NPR1 is translocated from the cytoplasm to the nucleus where it interacts with the transcription factor TGA2 to promote the expression of

\footnotetext{
* Correspondence: zhbwxd@hebau.edu.cn; wangxiaojie@nwsuaf.edu.cn 'State Key Laboratory of North China Crop Improvement and Regulation, College of Plant Protection, Technological Innovation Center for Biological Control of Crop Diseases and Insect Pests of Hebei Province, Hebei Agricultural University, Baoding, Hebei 071000, People's Republic of China ${ }^{3}$ State Key Laboratory of Crop Stress Biology for Arid Areas, College of Plant Protection, Northwest Agriculture and Forestry University, Yangling, Shaanxi 712100, People's Republic of China

Full list of author information is available at the end of the article
}

pathogenesis-related $(P R)$ genes (Cao et al. 1994; Delaney et al. 1995; Ryals et al. 1997; Shah et al. 1997; Mou et al. 2003). In Arabidopsis, NPR3 and NPR4 are paralogs of NPR1 that participate in CUL3 E3 ligase-mediated degradation of NPR1 in a SA concentration-dependent manner (Fu et al. 2012). Studies of the post-translational modification of NPR1 revealed complex regulation of this protein (Saleh et al. 2015; Withers and Dong 2016). NPR1 is a key regulator of defense across diverse plant species, making it a strong target gene for genetic engineering to improve disease and stress tolerance in crops (Silva et al. 2018; Backer et al. 2019). NPR1 may also be a target hub of effectors from various pathogens, including Pseudomonas syringae, Puccinia striiformis f. sp. tritici, and Phytophthora capsici (Wang et al. 2016; Chen et al. 2017; Li et al. 2019; Zhao et al. 2020).

The genes downstream of the plant defense response and SAR belong to 18 gene families, and are considered pathogenesis-related $(P R)$ genes that respond to various pathogens (van Loon et al. 2006; Ferreira et al. 2007; Sels et al. 2008; Wang et al. 2018). Among the PR genes characterized in wheat and barley, $P R 1$ is a hallmark of

(c) The Author(s). 2020 Open Access This article is licensed under a Creative Commons Attribution 4.0 International License, which permits use, sharing, adaptation, distribution and reproduction in any medium or format, as long as you give appropriate credit to the original author(s) and the source, provide a link to the Creative Commons licence, and indicate if changes were made. The images or other third party material in this article are included in the article's Creative Commons licence, unless indicated otherwise in a credit line to the material. If material is not included in the article's Creative Commons licence and your intended use is not permitted by statutory regulation or exceeds the permitted use, you will need to obtain permission directly from the copyright holder. To view a copy of this licence, visit http://creativecommons.org/licenses/by/4.0/. 
the activation of plant defense pathways (Molina et al. 1999) and is downstream of NPR1 in the acquired resistance triggered by Pseudomonas syringae pv. tomato DC3000 (Wang et al. 2016). The CAPE1 peptide derived from the C-terminal end of the PR1 protein is a damage-associated molecular pattern (DAMP) that triggers the accumulation of reactive oxygen species (ROS) and transcriptional activation of several $P R$ genes (Chen et al. 2014). The wheat TaPR1a protein can physically interact with ToxA to potentially mediate ToxA-induced necrosis in sensitive wheat lines ( $\mathrm{Lu}$ et al. 2014). A recent genetic and biochemical study showed that binding of PR1 to sterols can inhibit pathogen growth (Gamir et al. 2017). Interestingly, Arabidopsis AtPR1 can be targeted by a cerato-platanin protein, SsCP1 from Sclerotinia sclerotiorum, which suppresses the plant defense response (Yang et al. 2017).

Wheat rusts, including stripe rust, leaf rust, and stem rust, are severe fungal diseases that affect wheat crops (Hovmøller et al. 2010). The successful biotrophic lifestyle of rust fungi depends upon the ability of these fungi to deliver specialized effectors into host cells to suppress or evade plant defense. Recent genome sequencing approaches allow the prediction of hypothetical effectors from different wheat rust species (Duplessis et al. 2011; Cantu et al. 2013a; Zheng et al. 2013; Kiran et al. 2016; Zhao et al. 2020). The few well-characterized rust effector PST02549 from Puccinia striiformis f. sp. tritici (Pst) physically interacts with wheat EDC4 protein and is associated with processing bodies (Petre et al. 2016). Another wheat stripe rust effector, PEC6, inhibits pattern-triggered immunity (PTI) in a host species-independent manner and interacts with wheat adenosine kinases (Liu et al. 2016).

In our previous study, we used yeast two-hybrid screening to identify an effector protein PNPi from Pst that directly targets the wheat homolog of NPR1 protein (wNPR1)(Wang et al. 2016). PNPi interacts with the NPR1/NIM1-like domain of wNPR1 via its C-terminal DPBB_1 domain, and bimolecular fluorescence complementation (BiFC) assay revealed interaction between PNPi and wNPR1 in the nuclei of Nicotiana benthamiana protoplasts. In the same study, a transgenic barley line overexpressing the coding region of the signal peptide-truncated PNPi was generated to functionally characterize this effector. The induction of NPR1mediated $P R$ genes during SAR triggered by $P$. syringae DC3000 was suppressed by overexpression of PNPi, which might reflect a competitive effect of PNPi on the interaction between wNPR1 and wTGA2. The signal peptide of PNPi was sufficient to induce protein secretion from fungal yeast, but not from plant cells. Additionally, an RxLR-dEER motif-like region (RSLL-DEEP) of 24 amino acids was identified after the end of the predicted signal peptide.
In this investigation, we found that the wheat pathogenesis-related protein TaPR1a is targeted by PNPi in the apoplastic space. This interaction and TaPR1a function were further analyzed, and the results established the mechanism of a single rust fungal effector able to suppress multiple defense responses in wheat plants by targeting different components during infections. The generated transgenic wheat line TaPR1a-OE provides a valuable resource for the genetic improvement of wheat resistance to rust fungi.

\section{Results}

The wheat pathogenesis-related protein TaPR1a was targeted by the rust effector PNPi

A yeast two-hybrid $(\mathrm{Y} 2 \mathrm{H})$ library screening approach was used to identify proteins that interact with the rust effector PNPi (GenBank accession KT764125). The signal peptidetruncated PNPi was used as the bait, and the screen yielded 12 protein interactors (Additional file 1: Table S1). The gene expression patterns of these PNPi interactors during barley SAR-like response triggered by $P$. syringae DC3000 were examined using data from one of our previously established transcriptome databases (Additional file 2: Figure S1) (Gao et al. 2018). Since the expression level of the gene encoding barley pathogenesis-related protein HvPR1a_MLOC_52081 exhibited significant NPR1dependent up-regulation upon P. syringae DC3000 infection, we selected wheat TaPR1a (GenBank accession FJ815169) for further characterization as a PNPi interactor. Analysis of the TaPR1a sequence using SignalP 4.1 revealed a 24-amino acid signal peptide located at the $\mathrm{N}$-terminus of TaPR1a. To prevent the secretion of the tested proteins, we applied $\mathrm{Y} 2 \mathrm{H}$ validation using versions of these proteins lacking their respective signal peptides, $\mathrm{TaPR} \mathrm{a}_{(25-164)}$ and $\mathrm{PNPi}_{(23-333)}$. A strong interaction was observed between $\mathrm{TaPR} \mathrm{a}_{(25-164)}$ and $\mathrm{PNPi}_{(23-333)}$ on synthetic dropout (SD) selective medium lacking leucine, tryptophan, histidine, and adenine (SD-Leu-Trp-His-Ade) (Fig. 1a).

To determine the regions of PNPi required for its interaction with TaPR1a, two truncated PNPi proteins, $\operatorname{PNPi}_{(23-235)}$ and $\mathrm{PNPi}_{(236-333)}$, were prepared to examine their potential to interact with TaPR1a. $\mathrm{PNPi}_{(23-235)}$ contains the $\mathrm{N}$-terminal region of $\mathrm{PNPi}$ and $\mathrm{PNPi}_{(236-333)}$ contains the $\mathrm{C}$-terminal region of $\mathrm{PNPi}$, including the DPBB_1 domain (pfam03330) of PNPi. The results showed interactions of TaPR1a with both truncated PNPi proteins (Fig. 1a). We next cloned a homologous gene of PNPi from the wheat leaf rust pathogen, PtPNPi (Puccinia triticina PNPi homolog, GenBank accession OAV94594). Our initial $\mathrm{Y} 2 \mathrm{H}$ result indicated that the interactions were conserved between PtPNPi $(23-309)$ and wNPR1 (Genbank accession JX424315) or TaPR1a $a_{(25-164)}$ (Additional file 2: Figure S2). 


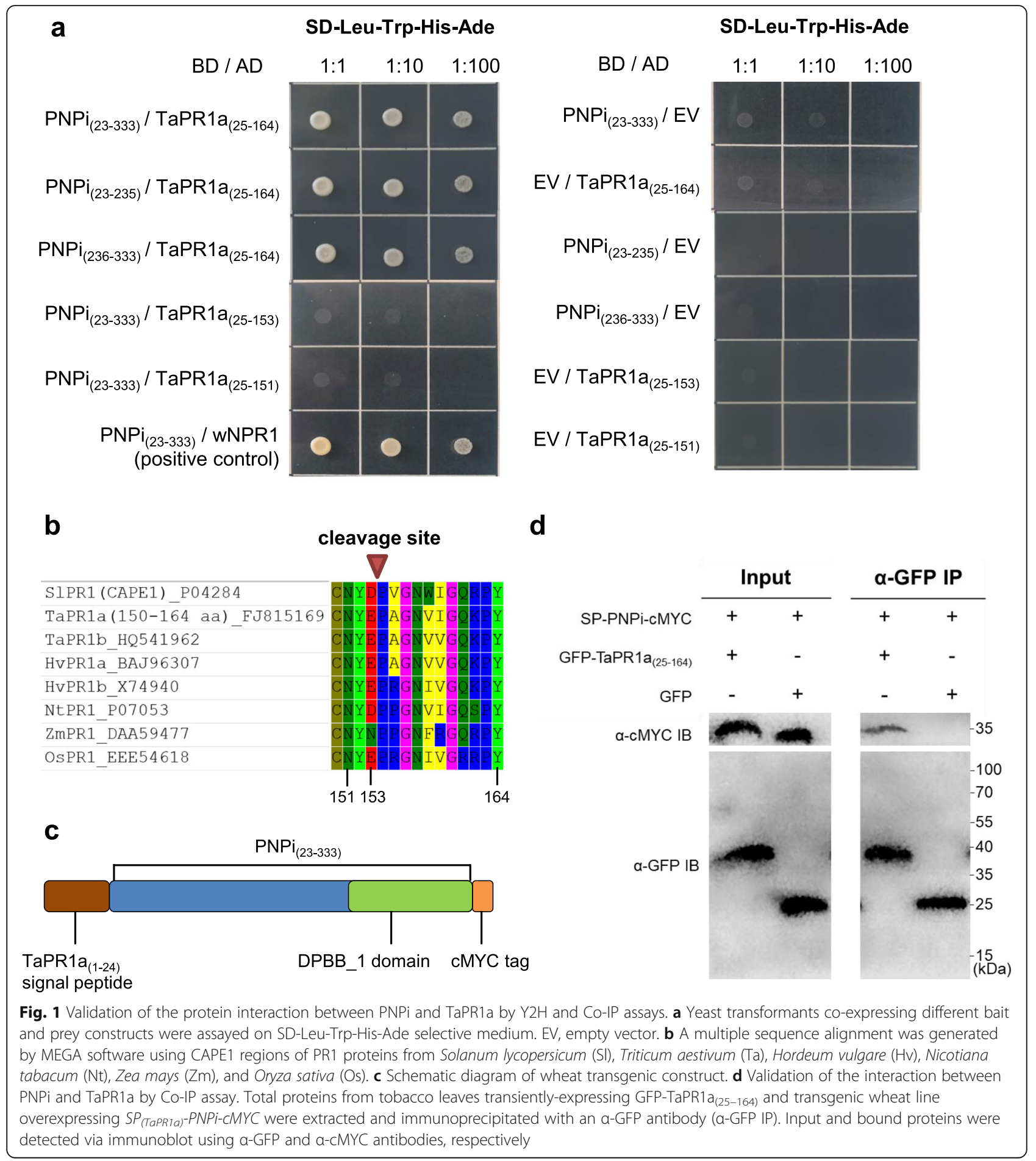

The C-terminal end of TaPR1a protein (CNYE_PAGNVIGQKPY) aligned well with the CAPE1 peptide found in most PR1 proteins (Fig. 1b). This peptide is responsible for the induction of CAPE1-mediated plant defense responses (Chen et al. 2014). To investigate whether the PNPi protein can interact with the CAPE1 region of TaPR1a, the coding regions of two CAPE1-truncated
TaPR1a proteins were constructed into the $\mathrm{Y} 2 \mathrm{H}$ vector as TaPR1a $a_{(25-153)}-A D$ and TaPR1a $a_{(25-151)}-A D$. The Y2H results showed that either truncation of the CAPE1 region in TaPR1a fully abolished interaction with PNPi (Fig. 1a).

The interaction between PNPi and TaPR1a was next validated by co-immunoprecipitation (Co-IP) assay. To do this, green florescent protein $(\mathrm{GFP})$ or GFP-TaPR1a $\mathrm{a}_{25-}$ 
164) was transiently expressed in tobacco leaves. Next, to promote the secretion of PNPi in wheat plant, we recombined the sequence encoding the signal peptide of TaPR1a and the signal peptide-truncated PNPi into our transgenic construct (Fig. 1c). Transgenic wheat lines overexpressing the recombinant transcript of $S P_{\text {(TaPRIa) }}$ PNPi-cMYC under the control of the maize Ubiquitin promoter $(S P$ PNPi-OE) were generated using Agrobacterium-mediated transformation in the spring common wheat cultivar "JW1". For the Co-IP assay, total proteins were extracted from Agrobacterium-infiltrated tobacco leaves and a transgenic wheat line overexpressing $S P_{\text {(TaPR1a) }}$-PNPi-cMYC. A specific signal for $\mathrm{SP}_{(\mathrm{TaPR} 1 \mathrm{a})}-\mathrm{PNPi}-\mathrm{cMYC}$ was clearly detected in the GFP-TaPR1a $a_{(25-164)}$ immunoprecipitate, but not in the GFP control (Fig. 1d), confirming the physical association of PNPi with TaPR1a.

\section{Protein interaction between PNPi and TaPR1a in the apoplastic space was validated in $N$. benthamiana} To validate the function of the signal peptide of TaPR1a, a red florescent protein (RFP) tag was fused to the Cterminus of TaPR1a. The RFP-fused protein was transiently expressed in $N$. benthamiana by Agrobacterium-mediated transformation. Next, plasmolysis was induced by addition of $800 \mathrm{mM}$ mannitol, and then clear apoplastic signals were observed for TaPR1a $\mathrm{a}_{(1-164)}$-RFP, but not for the empty vector (EV-RFP) control (Fig. 2a). To validate the secretion of the recombinant $\mathrm{SP}_{(\mathrm{TaPR} 1 \mathrm{a})}-\mathrm{PNPi}_{(23-333)}$, another construct of $\mathrm{SP}_{(\mathrm{TaPR} 1 \mathrm{a})}-\mathrm{PNPi}_{(23-333)}-\mathrm{GFP}$ was prepared and transiently expressed in tobacco leaves. Under plasmolysis treatment, dot-like apoplastic signals were observed for $\mathrm{SP}_{(\mathrm{TaPR} 1 \mathrm{a})}{ }^{-}$ $\mathrm{PNPi}_{(23-333)}$-GFP (Fig. 2b). Interestingly, we also observed GFP signals for the recombinant $\mathrm{SP}_{(\text {TaPR1a) }}-\mathrm{PNPi}_{(23-333)^{-}}$ GFP in both the cytoplasm and nucleus.

To further validate the interaction between PNPi and TaPR1a, we performed a bimolecular fluorescence complementation (BiFC) assay in $N$. benthamiana. Co-expression of $\mathrm{SP}_{(\mathrm{TaPR} 1 \mathrm{a})}-\mathrm{PNPi}_{(23-333)}-\mathrm{YFP}^{\mathrm{N}}$ and TaPR1a-YFP ${ }^{\mathrm{C}}$ generated yellow fluorescent protein (YFP) fluorescent signals in the apoplastic space (Fig. 3). Clear YFP fluorescent signals were also observed for the positive control, using AvrLm1YFP $^{\mathrm{N}}$ and BnMPK9-YFP ${ }^{\mathrm{C}}$ (Ma et al. 2018). Co-expression of each recombinant vector with the corresponding TaPR1a signal peptide-fused $\mathrm{YFP}^{\mathrm{N}}$ and $\mathrm{YFP}^{\mathrm{C}}$ vectors $\left(\mathrm{SP}_{(\mathrm{TaPR} 1 \mathrm{a})}{ }^{-}\right.$ $\mathrm{YFP}^{\mathrm{N}}$ and $\left.\mathrm{SP}_{(\mathrm{TaPR} 1 \mathrm{a})}\right)^{\left.-\mathrm{YFP}^{\mathrm{C}}\right)}$ served as negative controls, which showed no fluorescent signals.

\section{Transgenic wheat lines overexpressing TaPR1a showed} enhanced resistance to both $P s t$ and $P t$

To further explore the function of the TaPR1a gene, we generated transgenic wheat lines overexpressing TaPR1a in the spring common wheat cultivar "JW1". The expression levels of the overall endogenous and transgenic TaPR1a in the transgenic wheat line TaPR1a-OE ranged from 6.5- to 13.0-fold of that in wild-type plants (Fig. 4a). The third leaves of the transgenic wheat line TaPR1a-OE and wild-type plants were inoculated with urediniospores of the Pst pathotype CYR32, and CYR32 exhibited high virulence on wild-type plants (Fig. 4b). The susceptible phenotype (sporulation) in response to stripe rust pathogen infection was observed for all tested transgenic lines and wild-type plants. The percentage of Pst sporulation area for each inoculated leaf was calculated using ASSESS software (Additional file 2: Figure S3a). Compared with wild-type plants, significantly improved resistance to Pst was observed in TaPR $1 a-O E$ as evidenced by altered sporulation area $\left({ }^{*} P<0.05,{ }^{* *} P<0.01,6-10\right.$ biological replicates) (Fig. 4b and Additional file 1: Table S2).

Transgenic wheat line TaPR1a-OE and wild-type plants were next inoculated with urediniospores of the Pt pathotype THTT. A moderately susceptible phenotype (sporulation with necrosis) in response to leaf rust pathogen infection was observed in both TaPR1a-OE and wild-type plants (Fig. 4c). The percentage of $P t$ sporulation area in TaPR1a-OE was significantly lower ${ }^{* * *} P<0.01,16-39$ biological replicates) than that in wild-type plants (Fig. 4c, Additional file 1: Table S2, and Additional file 2: Figure S3b).

\section{Exogenous expression of $S P_{(T a P R 1 a)}-P N P i-C M Y C-O E$ in transgenic wheat lines reduced the degree of acquired resistance}

The successful establishment of the transgenic wheat line $S P_{(T a P R I a)}-P N P i-C M Y C-O E$ was confirmed by PCR of genomic DNA and RT-qPCR. The expression levels of the $S P_{(\text {TaPRIa) }}-P N P i-c M Y C$ transgene ranged from 24.2- to 80.5-fold of the levels of the endogenous control gene, TaActin (GenBank accession AB181991) (Fig. 5a). We did not detect definite signals corresponding to the $S P_{\text {(TaPR1a) }}$-PNPi-cMYC transcript in wild-type plants. $P$. syringae DC3000 was injected into wheat leaves to induce a SAR-like response (acquired resistance). To evaluate the degree of the acquired resistance, $M$. oryzae isolate P131 was inoculated adjacent to the $P$. syringae DC3000 infiltration area as the secondary pathogen. This inoculation was performed at 48 hours post-infiltration (hpi), when a clear cell death phenotype caused by $P$. syringae DC3000 was observed in the infiltration area (Fig. 5b). In wild-type plants, we observed significantly $\left({ }^{* *} P=0.009\right)$ improved resistance to $M$. oryzae isolate P131 in the region adjacent to the P. syringae DC3000 infiltration area, whereas the acquired resistance response was consistently suppressed in two independent transgenic wheat lines of SP-PNPi-OE (Fig. 5c and Additional file 1: Table S3). We further evaluated the acquired resistance to $M$. oryzae isolate P131 in transgenic wheat line TaPR1a-OE, but did not observe any differences between TaPR1a-OE and wild-type plants. 


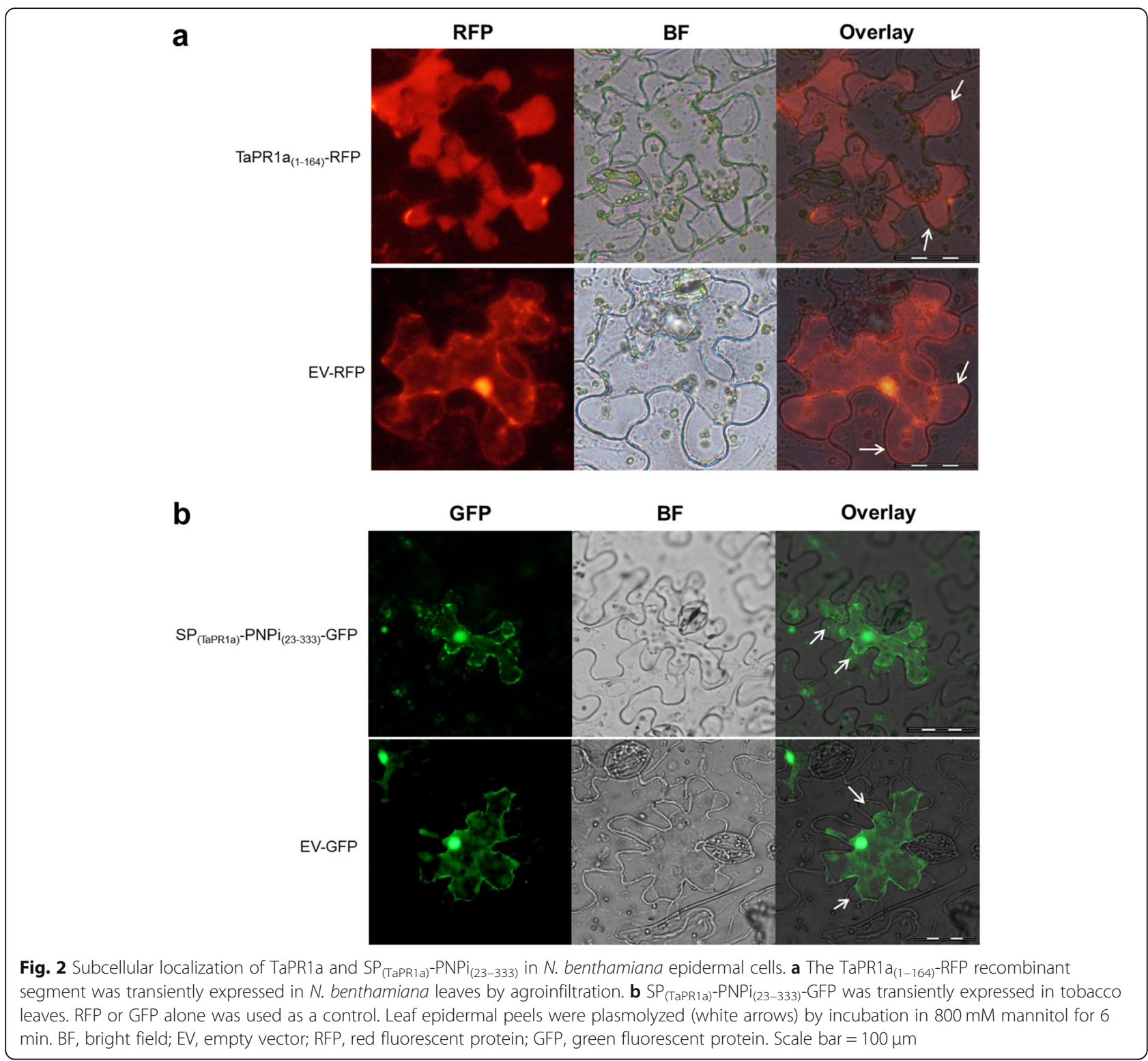

We also monitored the expression levels of several downstream $P R$ genes of NPR1-mediated acquired resistance, including wheat homologs of $P R 1 a, P R 2$, and $P R 4 b$ (GenBank accessions are listed in Additional file 1: Table S4), in part of the leaf adjacent to the P. syringae DC3000 infiltration area at 48 hpi by RT-qPCR assay (Fig. 5d). Samples from the region adjacent to the water-infiltration area were employed as controls. P. syringae DC3000 infection significantly elevated the expression levels of TaPR1a ( $\left.{ }^{* * *} P<0.0001\right)$, TaPR2 ( $\left.{ }^{* *} P=0.0031\right)$, and TaPR4b ( $P=$ 0.0127) (Fig. 5d and Additional file 1: Table S5). Compared with the expression levels in wild-type plants, the expression levels of TaPR1a ( $\left.{ }^{* *} P=0.0017\right), T a P R 2(* * P=$ $0.0012)$, and TaPR4b (**P=0.0023) induced by $P$. syringae DC3000 infection were significantly suppressed in two independent transgenic wheat lines of SP-PNPi-OE (Fig. 5d).
We also examined the possible function of the TaPR1a gene during the acquired resistance triggered by $P$. syringae DC3000. To do this, RT-qPCR assay was employed to evaluate the expression levels of TaPR2 and TaPR4b in the adjacent region of $P$. syringae DC3000 infection in the transgenic wheat line TaPR1a-OE and wild-type plants. However, we did not detect any significant differences in the expression levels of these two genes between the transgenic line and wild-type plants (Additional file 1: Table S5 and Additional file 2: Figure S4).

Expression levels of TaAdi3 and TaPR7 were suppressed in the transgenic wheat line SP-PNPi-OE

Several genes were previously reported as induced by chemically synthesized CAPE1 peptide in tomato plants (Chen et al. 2014), including PI-1 (proteinase inhibitor 1, 


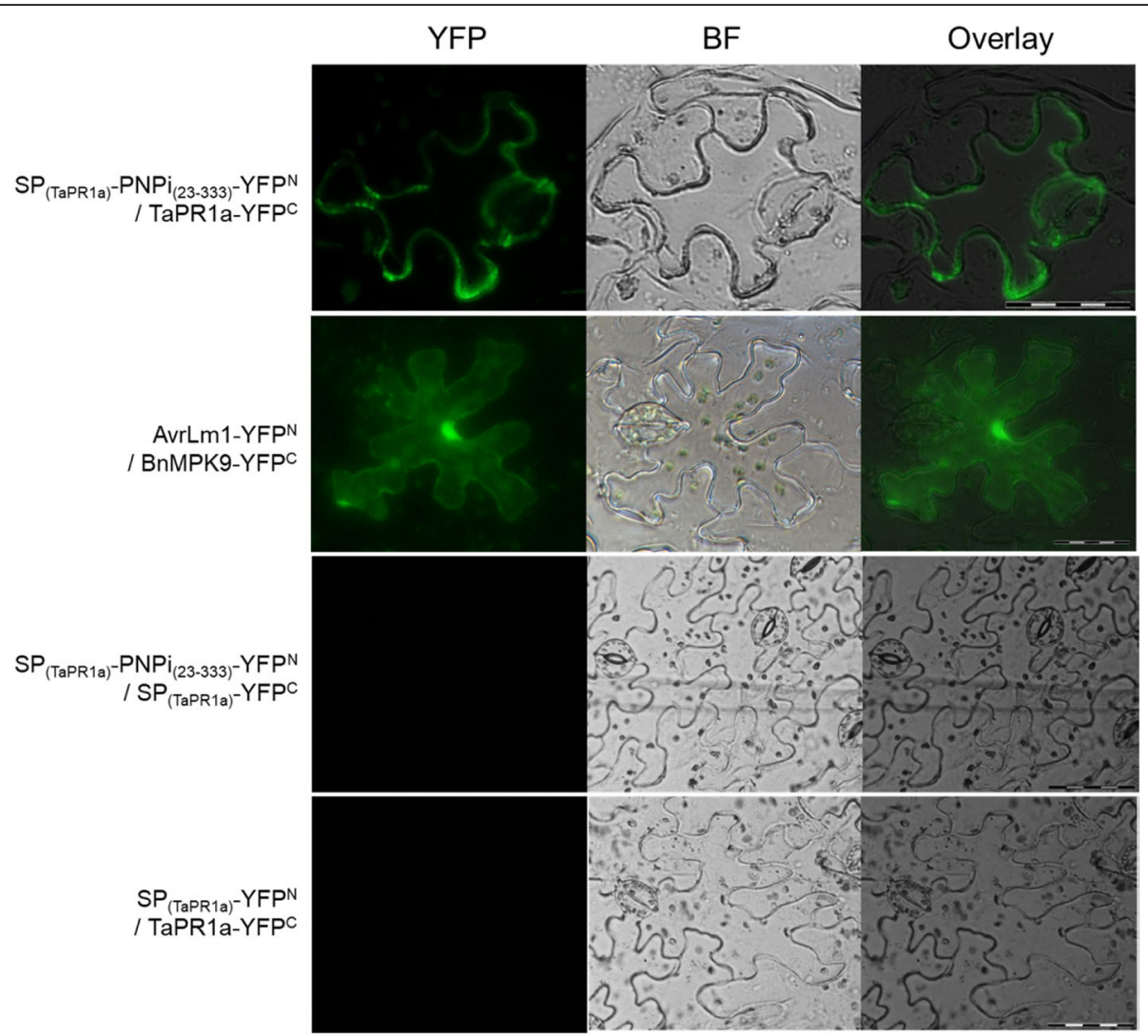

Fig. 3 The interaction of PNPi and TaPR1a proteins was detected by BiFC assay. YFP signals were detected in the leaves of N. benthamiana coexpressing $\mathrm{SP}_{(\mathrm{TaPR} 1 \mathrm{a})}-\mathrm{PNPi}_{(23-333)^{-}} \mathrm{YFP}^{\mathrm{N}}$ and TaPR1a-YFPC . Co-expression of AvrLm1-YFP ${ }^{\mathrm{N}}$ and BnMPK9-YFPC was utilized as a positive control. Coexpression of each recombinant vector with the corresponding TaPR1a signal peptide-fused YFP ${ }^{N}$ and YFPC vectors served as negative controls. $\mathrm{BF}$, bright field; EV, empty vector; YFP, yellow fluorescent protein. Scale bar $=100 \mu \mathrm{m}$

also known as BCI7, GenBank accession K03290), PI-2 (proteinase inhibitor 2, K03291), Adi3 (AvrPto-dependent Pto-interacting protein 3, NM_001247682), ERF5 (ethylene response factor 5, NM_001247583), PR-1b (pathogenesisrelated protein 1b, M69248.1), PR-2 (beta-1,3-glucanase, NM_001247869.1), PR-7 (Cys protease, CK574973.1), and Chit2;1 (Class II chitinase, U30465.1). We tried to collect expression data for the closest homologs of these genes in wheat and barley, but did not obtain data for PI-2 (no homologs) and ERF5 (GenBank accession removed). Interestingly, $H v P I-1 / H v B C I 7, H v P R 1 b, H v P R 2$, and HvChit $2 a$ in barley were previously designated as genes downstream of NPR1 and highly expressed during acquired resistance triggered by P. syringae DC3000 (Gao et al. 2018). The expression data indicated that HvAdi3 (GenBank accession AK355246) and HvPR7 (BAK05599) were neither involved in acquired resistance nor regulated by NPR1 (Additional file 2: Figure S5). Thus, homologs of Adi3 and PR7 in wheat may be suitable candidates to study the influence of PNPi on TaPR1a independently of wNPR1.

We used P. syringae DC3000 to trigger the plant defense response and performed RT-qPCR assay to monitor the expression levels of TaAdi3 (GenBank accession XM_
020291623) and TaPR7 (XP_020149865) in transgenic wheat lines TaPR1a-OE and SP-PNPi-OE in the local infiltration region at $6 \mathrm{hpi}$. Expression levels of both TaAdi3 $\left({ }^{* *} P=0.0005,6-8\right.$ biological replicates) and TaPR7 $(* * P=$ 0.0002 ) were significantly induced by $P$. syringae DC3000 infection in wild-type plants and increased to even higher levels $\left.{ }^{* *} P<0.01\right)$ in the transgenic wheat line TaPR1a-OE (Fig. 6 and Additional file 1: Table S5). Compared with wild-type plants, we detected significant decreased induction of TaAdi3 $(* P=0.0002)$ and TaPR7 $(* * P=0.0012)$ in the transgenic wheat line SP-PNPi-OE (Fig. 6).

\section{Discussion}

In our previous study, we discovered a virulent rust effector PNPi that can suppress systemic acquired resistance by directly targeting the wheat NPR1 protein (Wang et al. 2016). In this work, using a classic $\mathrm{Y} 2 \mathrm{H}$ library screening approach, we identified 12 potential PNPi interactors (Additional file 1: Table S1). Based on the expression patterns of these interactor-encoding genes during SAR-like response triggered by $P$. syringae DC3000 in barley (Additional file 2: Figure S1), we selected TaPR1a, a wheat pathogenesisrelated protein as a putative interactor of PNPi for further 

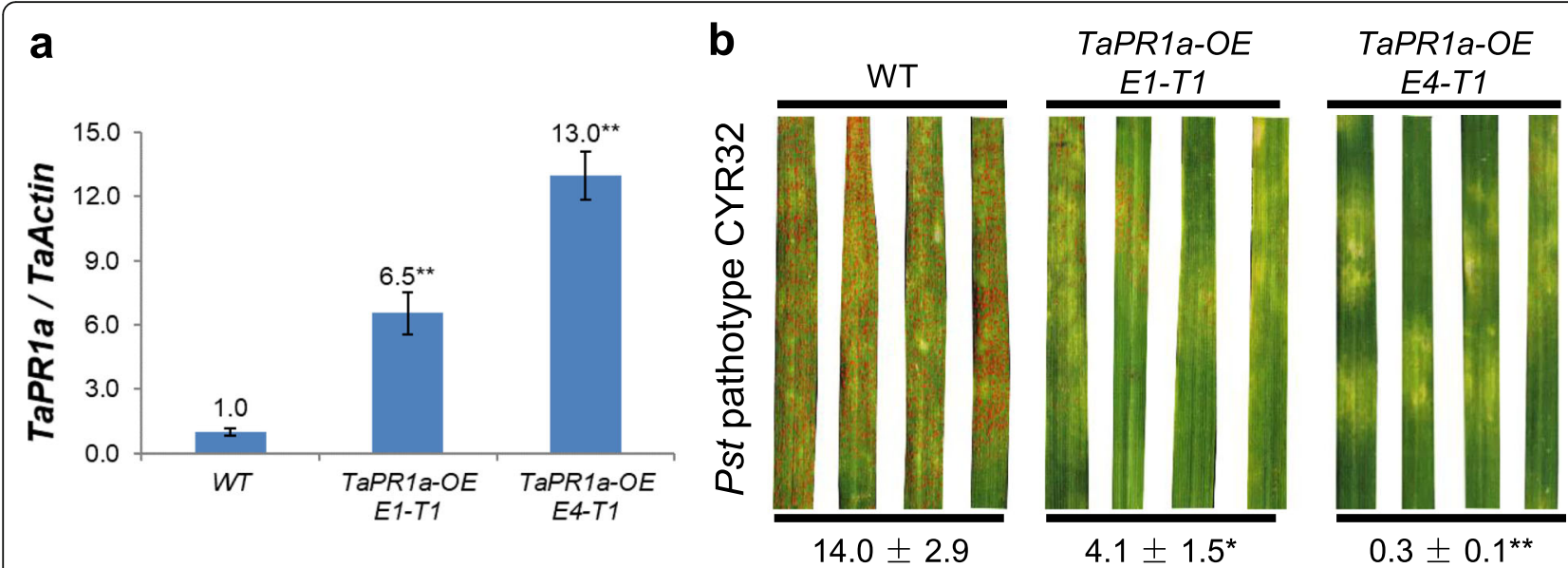

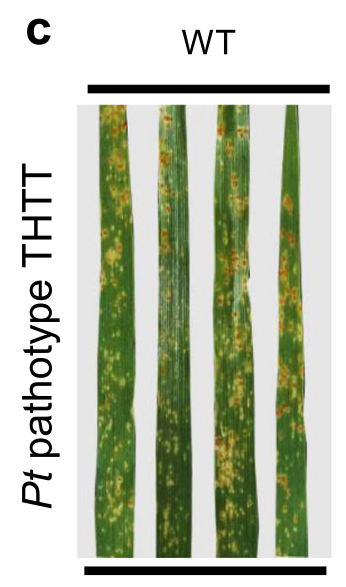

$3.2 \pm 0.7$
TaPR1a-OE $\mathrm{E} 1-\mathrm{T} 1$

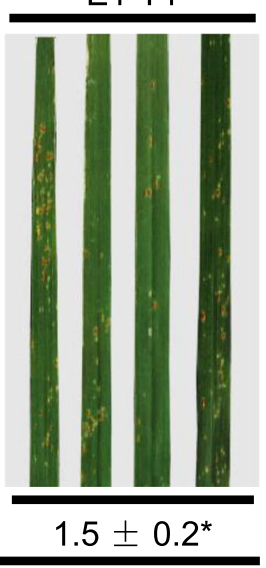

$1 s t$

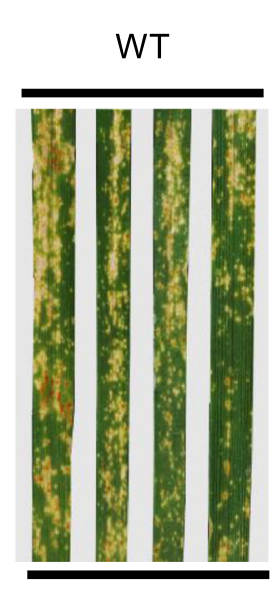

$5.9 \pm 1.1$

$4.1 \pm 1.5^{\star}$

$0.3 \pm 0.1^{* *}$

TaPR1a-OE E4-T1

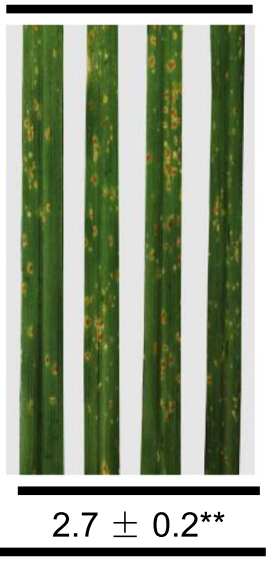

\section{$2 n d$}

Fig. 4 Transgenic wheat line TaPR1a-OE showed enhanced resistance to both Pst and Pt. a The transcript levels of TaPR1a in TaPR1a-OE were determined by RT-qPCR. b The phenotypes of TaPR1a-OE and wild-type plants in response to the highly virulent Pst pathotype CYR32. c The susceptibility phenotypes of TAPR1a-OE and wild-type plants to the Pt pathotype THTT. The numbers below the images of the leaves represent the average percentages of the rust sporulation area in each inoculated leaf. The asterisks $\left({ }^{*} P<0.05,{ }^{* *} P<0.01\right)$ indicate the significance of the differences between the transgenic line and wild-type plants as assayed by Dunnett's test

characterization. The physical interaction between PNPi and TaPR1a was further validated by Co-IP assay (Fig. 1d). In our previous investigation, we determined the DPBB_1 domain of PNPi was required for interaction with wNPR1 (Wang et al. 2016). However, we did not identify specific domains in PNPi responsible for interaction with TaPR1a (Fig. 1a). The observed interactions of PtPNPi (homolog of PNPi in $P t$ ) with both wNPR1 and TaPR1a by $\mathrm{Y} 2 \mathrm{H}$ assay (Additional file 2: Figure S2) suggest potential conserved features among PNPi homologs from different rust species.

An RXLR-DEER-like (RSLL-DEEP) region was identified in the $\mathrm{N}$-terminal region of the PNPi protein after the signal peptide, but the translocation mechanism of this rust effector from the apoplastic space to the cytoplasm and nucleus was not previously characterized. Under plasmolysis condition, apoplastic signals were observed for both TaPR1a-RFP and $\left.\mathrm{SP}_{(\text {TaPR1a) }}-\mathrm{PNPi}_{(23-333)}\right)^{-G F P}$ in $N$. benthamiana (Fig. 2). In a recent investigation, a similar apoplastic localization of TaPR1a-GFP was observed in tobacco leaves under plasmolysis treatment (Wang et al. 2020). We also observed cytoplasmic and nuclear signals for the recombinant $\mathrm{SP}_{(\mathrm{TaPR} 1 \mathrm{a})}-\mathrm{PNPi}_{(23-333)}-\mathrm{GFP}$ protein, which might be caused by the cell re-entry process of the PNPi protein via its N-terminal RSLL-DEEP region, or could be generated by traffic congestion of the secretion pathway in the Agrobacterium-mediated transient expression assay (Wawra et al. 2017). In a previous study, transiently expressed TaPR1-GFP (approximately $40 \mathrm{kDa}$ in size) in tobacco leaves was very stable, with no cleavage of the C-terminal CAPE1 region (Breen et al. 2016). Thus, we speculated that cleavage of CAPE1 did not occur on the time scale of our transient expression of GFP${\text { TaPR } 1 a_{(25-164)} \text { and TaPR1a-YFP }}^{C}$ in the Co-IP and BiFC assays. Based on the detection of recombined YFP signals 


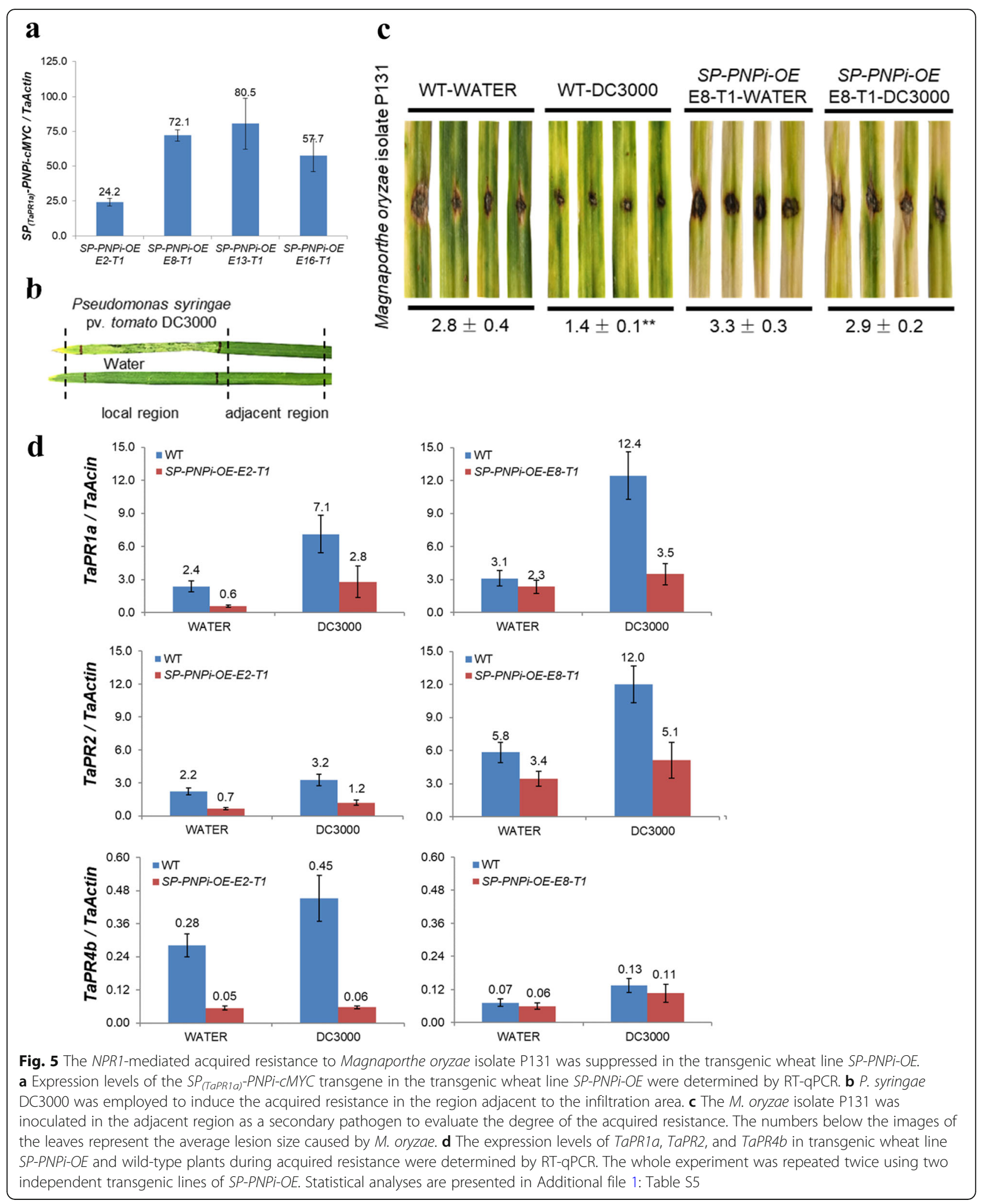



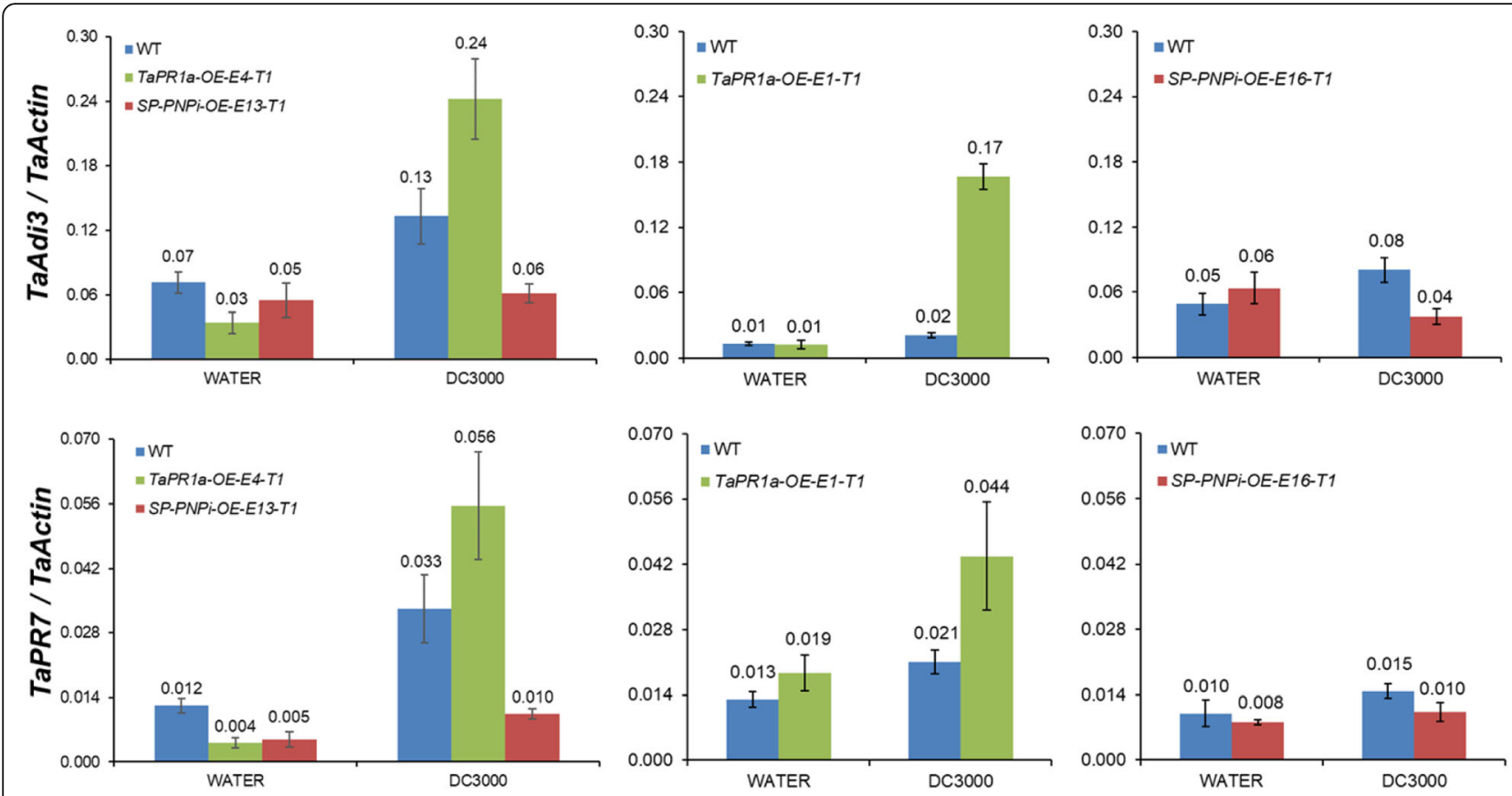

Fig. 6 The transcriptional levels of TaAdi3 and TaPR7 were determined by RT-qPCR in transgenic wheat lines TaPRTa-OE and SP-PNPi-OE upon P. syringae DC3000 infection. Two independent transgenic lines of TaPR1a-OE and SP-PNPi-OE were tested. Statistical analyses are presented in Additional file 1: Table S5

in $N$. benthamiana co-expressing $\mathrm{SP}_{(\mathrm{TaPR} 1 \mathrm{a})} \mathrm{PNPi}_{(23-333)^{-}}$ $\mathrm{YFP}^{\mathrm{N}}$ and TaPR1a-YFP ${ }^{\mathrm{C}}$ (Fig. 3), we predicted interation of the rust effector PNPi with wheat TaPR1a in the apoplastic space.

PR1 family proteins have been reported to be involved in various biological processes, including sterol binding, defense signaling, and as targeting hubs of pathogen effectors (Breen et al. 2017). Resistance to both Pst and Pt was significantly improved in the transgenic wheat line TaPR1a-OE (Fig. 4 and Additional file 1: Table S2). Although we detected no high-resistance phenotypes in TaPR1a-OE, the significantly diminished or delayed infection processes of both the virulent Pst pathotype CYR32 and the Pt pathotype THTT were observed. Therefore, the generated transgenic wheat line TaPR1a-OE provides a valuable resource for future efforts to improve wheat resistance to rust diseases.

In our previous investigation, we validated the role of PNPi in the suppression of SAR using transgenic barley line overexpressing the transcript that encodes the signal peptide-truncated $\mathrm{PNPi}_{(23-333)}$ (Wang et al. 2016). Previous work showed that the signal peptide of PNPi can guide the secretion of a protein from an yeast fungus, but not from a plant cell (Wang et al. 2016). To confirm the function of $P N P i$ in the natural wheat host and imitate the apoplastic state of this rust effector, we developed a transgenic wheat line overexpressing the recombinant transcript of $S P_{(\mathrm{Ta}-}$ PR1a)-PNPi-cMYC. The model bacterial pathogen P. syringae DC3000 and the fungal pathogen $M$. oryzae isolate
P131 were applied as an inducer and a secondary invader, respectively, to evaluate the degree of NPR1-mediated acquired resistance in wheat plants (Colebrook et al. 2012; Wang et al. 2016; Gao et al. 2018). Reduced acquired resistance to $M$. oryzae isolate P131 was observed in the transgenic wheat line SP-PNPi-OE, with significant suppression of TaPR1a, TaPR2, and TaPR4b genes (Fig. 5 and Additional file 1: Table S3). These findings were similar to those we observed previously in the HVNPR1-silenced transgenic barley line (Gao et al. 2018).

The CAPE1 peptide of PR1 protein was reported as a DAMP during plant immune response that could act to transcriptionally activate several $P R$ genes (Chen et al. 2014). RT-qPCR results confirmed that the observed increases of TaAdi3 and TaPR7 genes were directly associated with the increased expression level of TaPR1a transgene in the local region inoculated with $P$. syringae DC3000 (Fig. 6). These results were consistent with those of a previous study in which the chemically synthesized CAPE1 peptide was applied as an inducer of $P R$ genes in tomato plants (Chen et al. 2014). Regulation of the TaAdi3 and TaPR7 genes may independent of NPR1 based on our previous transcriptome data (Additional file 2: Figure S5) (Gao et al. 2018), making these genes ideal candidates for further investigation of the wNPR1independent effect of PNPi on TaPR1a. The detected significant suppression of TaAdi3 and TaPR7 in the transgenic wheat line SP-PNPi-OE (Fig. 6), together with the evidence of the requirement of the CAPE1 region for 
interaction between PNPi and TaPR1a (Fig. 1a) provide valuable clues to help elucidate the possible working model of the rust effector PNPi.

\section{Conclusions}

During the early stage of infection, rust fungus induces the cleavage of the conserved CAPE1 peptide from the Cterminal end of the TaPR1a protein, allowing recognition by a pattern recognition receptor (PRR). The signal will be transduced to eventually drive transcriptional activation of TaAdi3 and TaPR7 genes. In the apoplastic space or extrahaustorial matrix, rust fungus secretes the effector PNPi to target the CAPE1 region of the TaPR1a protein, and this interaction may suppress the expression of TaAdi3 and TaPR7. PNPi can be translocated into the plant cell and nucleus to suppress the expression burst of $P R$ genes by directly targeting wheat NPR1.

\section{Methods}

\section{Screening of $\mathrm{Y} 2 \mathrm{H}$ library using PNPi as a bait}

The $\mathrm{Y} 2 \mathrm{H}$ cDNA library tested here was previously constructed from Puccinia striiformis f. sp. tritici-infected leaves of the Triticum turgidum subsp. durum cultivar "Langdon" (line RSL65) (Yang et al. 2013). The wheat line RSL65 carries the Yr36 resistant gene, which confers partial resistance to Pst at temperature higher than $25^{\circ} \mathrm{C}(\mathrm{Fu}$ et al. 2009). Seedlings of RSL65 were grown in a growth chamber with a nocturnal temperature of $10^{\circ} \mathrm{C}$, a diurnal temperature of $15^{\circ} \mathrm{C}$, and $16 \mathrm{~h}$ of light. Under these conditions, Yr36 resistance is not active, so plants are partially susceptible to Pst. RSL65 seedlings were inoculated with urediniospores of Pst pathotype PST-133 as previously described (Fu et al. 2009). Pst-infected leaves were sampled at $0,3,9$, and 15 days post-inoculation (dpi). Total RNA were extracted from samples using TRIzol reagent (Invitrogen, Carlsbad, CA, USA). Extracted RNAs were pooled and reverse-transcribed into cDNA, using the Make Your Own "Mate \& Plate" Library System following the manufacturer's protocol (Clontech, Mountain View, CA, USA). The resulting cDNAs were then recombined into the prey vector (pGADT7Rec) using Clontech's SMART technology, and the final library was transformed into the yeast strain Y187. Next, the transcript encoding $\operatorname{PNPi}_{(23-333)}$ was cloned into the bait vector pLAW10 (BD) and introduced into the yeast strain "Y2H Gold" using the lithium acetate method (Cantu et al. 2013b). The pLAW10$\operatorname{PNPi}_{(23-333)}$ construct was tested against an empty vector and was not auto-activated on SD-Leu-Trp-His-Ade. The bait colonies of pLAW10-PNPi $\mathrm{P}_{(23-333)}$ were grown to approximately $10^{8} \mathrm{cfu} / \mathrm{mL}$ in $50 \mathrm{~mL}$ SD-Trp liquid medium. Yeast cells were pelleted, washed once with sterile $\mathrm{H}_{2} \mathrm{O}$, and re-suspended in $50 \mathrm{~mL}$ of liquid media consisting of $2 \times$ YPDA. One aliquot of the Y187 target yeast $\left(>2 \times 10^{7}\right.$ cells) was mixed with the bait. After incubation at $30^{\circ} \mathrm{C}$ for $20-24 \mathrm{~h}$ with slight shaking, the yeast cells were isolated, washed twice with sterile water, and then plated on SD-Leu-Trp-His-Ade medium. Yeast putative positive diploids from primary screenings were isolated and plasmids were extracted using Zymoprep I ${ }^{\mathrm{TM}}$ Yeast Plasmid Minipreparation Kit (Zymo Research, CA, USA). Matchmaker AD primers were used to amplify the inserted gene fragments. Sequence annotations were performed with BLASTx homology searches against the NCBI GenBank nr database (https://www.ncbi.nlm.nih.gov/).

\section{$\mathrm{Y} 2 \mathrm{H}$ validation}

Primers were designed to amplify the coding region of TaPR1a (Additional file 1: Table S4). The complete coding region of TaPR1a was amplified from cDNA synthesized from RNA isolated from the seedlings of the common wheat line "Chinese Spring". SignalP 4.1 server (http:// www.cbs.dtu.dk/services/SignalP/) was employed to identify the signal peptide. The coding regions of the signal peptide-truncated PNPi and TaPR1a were cloned into Gateway Y2H vectors pLAW10 (BD) and pLAW11 (AD), respectively. Recombinant $\mathrm{Y} 2 \mathrm{H}$ vectors carrying different regions of the $P N P i$ gene were constructed and described in our previous study (Wang et al. 2016). The coding sequence of PNPi (23-235) started immediately after the end of the 22-amino-acid-long predicted signal peptide and included 213 amino acids from the N-terminal region of the PNPi protein. PNPi (236-333) included the DPBB_1 domain located in the C-terminal region of the protein. TaPR1a $a_{(25-164)}$ encoded the signal peptide-truncated TaPR1a. TaPR1a $a_{(25-153)}$ and TaPR1a $a_{(25-151)}$ are derivatives of TaPR1a with both the signal peptide and the C-terminal CAPE1 peptide region truncated. Co-transformed yeast strains were assayed on plates of SD-Leu-Trp-His-Ade selective medium.

We cloned the coding region of PtPNPi using cDNA synthesized from RNA isolated from wheat seedlings of the common wheat line "Chinese Spring" that were inoculated with Pt pathotype PHTT(P) (Zhao et al. 2020). The coding region of the signal peptide-truncated PtPNPi was cloned into Gateway Y2H vector pLAW11 (AD). The wNPR1-BD construct was constructed in our previous study (Cantu et al. 2013b). The co-transformed yeast strains were assayed on selective medium (SD-LeuTrp-His-Ade).

\section{Subcellular localization and BiFC}

To confirm the signal peptide of TaPR1a predicted by the SignalP 4.1, a RFP tag was fused to the C-terminus of TaPR1a and a GFP tag was fused to the C-terminal end of the recombinant $\mathrm{SP}_{(\mathrm{TaPR} 1 \mathrm{a})}-\mathrm{PNPi}_{(23-333)}$ protein. The resulting constructs were transformed into the Agrobacterium strain GV3101, and then used to infiltrate four-week-old $N$. benthamiana plants. An empty 
pGWB5 vector expressing only GFP and an empty pGWB454 vector expressing only RFP served as controls. Green or red fluorescence was detected $48 \mathrm{hpi}$ using a Nikon Ti-2 fluorescence microscope with a filter specific to GFP or RFP, respectively. Epidermal peels from $N$. benthamiana leaves were plasmolyzed in 800 $\mathrm{mM}$ mannitol for $6 \mathrm{~min}$.

BiFC assay was conducted using a split YFP system in $N$. benthamiana leaves, as previously described ( $\mathrm{Ma}$ et al. 2018). The complete coding regions of TaPR1a, as well as the recombinant transcript of $S P_{(\text {TaPRIa) }}-P N P i_{(23-333)}$, were fused to the $\mathrm{N}$ - and $\mathrm{C}$-terminal regions of YFP in Gateway destination vectors $\mathrm{pDEST}^{\mathrm{GW}}{ }^{\mathrm{GYNE}}$ and $\mathrm{pDEST}-{ }^{\mathrm{GW}-}$ VYCE, respectively. The fusion proteins were then coexpressed in $N$. benthamiana leaves using Agrobacteriummediated transformation. Fluorescence was monitored approximately $48 \mathrm{hpi}$, using a Nikon fluorescence microscope with a YFP filter. Co-transformation of AvrLm1-YFP ${ }^{\mathrm{N}}$ and BnMPK9-YFP ${ }^{\mathrm{C}}$ was utilized as a positive control (Ma et al. 2018). Signal peptide-fused empty vectors of $\mathrm{SP}_{(\mathrm{TaPR} 1 \mathrm{a})^{-}}$ $\mathrm{YFP}^{\mathrm{N}}$ and $\mathrm{SP}_{(\mathrm{TaPR} 1 \mathrm{a})}-\mathrm{YFP}^{\mathrm{C}}$ were used as negative controls.

\section{Co-IP assay}

The coding sequence of the signal peptide-truncated TaPR1a was cloned into the Gateway compatible GFPtagged expression vector pMDC43 (Curtis and Grossniklaus 2003) as 35S::GFP-TaPR1a $a_{(25-164)}$. Tobacco leaves were infiltrated with Agrobacterium carrying 35S::GFP-TaPR1a $a_{(25-}$ 164), and infiltration with 35S::GFP was performed as a control. Protein samples were extracted from Agrobacteriuminfiltrated tobacco leaves at $3 \mathrm{dpi}$ and the transgenic wheat line overexpressing $S P_{\text {(TaPRIa) }}$-PNPi-cMYC. Next, $\alpha$-GFP IP was performed using GFP-trap beads (LABLEAD, Beijing, China) as previously described (Du et al. 2015). Total proteins and immunoprecipitates were separated by SDSPAGE, and then detected by immunoblots using $\alpha$-GFP antibody (Solarbio, Beijing, China) or $\alpha$-cMYC antibody (Solarbio, Beijing, China). Original, uncropped blots are provided as Additional file 2: Figure S6.

\section{Plant materials and induction of acquired resistance}

All transgenic wheat lines used in this study were generated in our lab with technical help from Jinan Bangdi Ltd. Company, Shandong, China. The transgenic background material "JW1" was selected by this company from a segregating population of the cross of spring common wheat cultivars "Fielder" and "NB1". Briefly, the full-length ORF of TaPR1a and the recombinant transcript of $S P_{\text {(TaPRIa) }^{-}}$ PNPi-cMYC (PNPi with the signal peptide of TaPR1a and a C-terminal cMYC tag), were cloned into a wheat transgenic vector, pLGY-02, that contained the maize (Zea mays) ubiquitin 1 promoter and T-DNA insertion site. Transgenic wheat lines were generated using Agrobacterium-mediated transformation.
Acquired resistance was induced by inoculation with $P$. syringae DC3000, as previously described (Colebrook et al. 2012). Briefly, P. syringae DC3000 was grown on King's B medium supplemented with $50 \mu \mathrm{g} / \mathrm{mL}$ rifampicin antibiotics and then diluted to OD600 $=0.6$ in sterile water. Third leaves of transgenic wheat lines SP-PNPi$O E$ and TaPR1a-OE as well as wild-type plants were inoculated using a $1-\mathrm{mL}$ needless syringe by pressure infiltration of bacterial suspensions through the leaf abaxial surface. The borders of each infiltrated region were marked using a marker pen and control seedlings were similarly infiltrated with sterile water. After bacterial inoculation, the seedlings were transferred under a constant temperature of $23^{\circ} \mathrm{C}$ to facilitate bacterial growth. Samples for RT-qPCR assay were collected from regions adjacent to the infiltration area (approximately $1 \mathrm{~cm}$ from the border of the infiltrated area) at $48 \mathrm{hpi}$.

$M$. oryzae inoculation was performed on adjacent regions of leaves to evaluate the degree of acquired resistance in the transgenic wheat line SP-PNPi-OE and wildtype plants. The $M$. oryzae isolate $\mathrm{P} 131$ was grown on tomato-oat medium for $11 \mathrm{~d}$ at $25^{\circ} \mathrm{C}$ under a $16 / 8 \mathrm{~h}$ light-dark cycle. Ten $\mathrm{mL}$ of the P131 conidia suspension $\left(5.0 \times 10^{5}\right.$ spores $\left./ \mathrm{mL}\right)$ containing $0.05 \%$ Tween-20 was dropped onto press-injured spots in adjacent region. The plants were then incubated in a mist chamber at $25^{\circ} \mathrm{C}$ in the dark for the first 24 hpi before transfer to a growth chamber at $23^{\circ} \mathrm{C}$ and $80 \%$ humidity under a photoperiod of $16 \mathrm{~h}$ light and $8 \mathrm{~h}$ dark. The inoculated leaves were photographed 5 days later and the size of the lesion caused by P131 on each leaf was measured. Six to ten biological replicates were performed for each treatment and phenotype combination, and the whole experiment was repeated twice using two independent transgenic lines. Dunnett's test was performed using SAS software v9.4 (SAS Institute, Cary, NC, USA).

\section{Wheat rust inoculation}

Seedlings of the transgenic wheat line TaPR1a-OE and wild-type plants were maintained and inoculated with Pst pathotype CYR32 and Pt pathotype THTT according to the previously described procedures and conditions (Huang et al. 2019; Wu et al. 2019). The fully expanded third leaves of wheat seedlings were spray-inoculated with uredospores of CYR32 and THTT in water solution. The disease symptoms were scored at $14 \mathrm{dpi}$. The percentages of the Pst or Pt sporulation area for each inoculated leaf were determined using ASSESS image analysis software v2.0 for plant disease quantification (Lamari 2008). This software was previously applied for evaluation of wheat resistance to stem rust (Zhang et al. 2017), stripe rust, and powdery mildew (Li et al. 2020). The ASSESS software recognizes rust uredinium in an inoculated leaf based on individual pixel color (Additional file 2: Figure S3). For Pst 
inoculation, two independent transgenic lines were employed with 6-10 biological replicates. Data were transformed for normality restoration, and Dunnett's test was performed using SAS software v9.4 (SAS Institute, Cary, NC, USA). For Pt inoculation, the whole experiment was repeated twice using two independent transgenic lines. Each experiment of 16-39 biological replicates was considered a block. The data were log-transformed to restore normality, and two-sample t-test was conducted using SAS software v9.4.

\section{RNA extraction and RT-qPCR}

Total RNA samples were prepared by extraction using a RNA Extraction kit (QIAGEN, Hilden, Germany) in accordance with the manufacturer's instructions, and then first-strand cDNA samples were synthesized using a Reverse Transcription kit (Takara, Dalian, China). Equal amounts of RNA were used for each reverse transcription reaction. The primers utilized for RT-qPCR assay are listed in Additional file 1: Table S4. The wheat TaActin gene (GenBank accession AB181991) was employed as an internal reference for the analyses (Paolacci et al. 2009). The amplification efficiency of each pair of primers was calculated by a preliminary RT-qPCR assay using six 2 -fold diluted cDNA samples (1:1, 1:2, 1:4, 1:8, 1:16, and 1:32). Next, dissociation curves were generated at temperatures ranging from 60 to $94{ }^{\circ} \mathrm{C}$ to ensure that a single and specific product was amplified. Gene expression was quantified using the threshold values $(\mathrm{Ct})$ generated by the Roche LightCycler 96 (Roche, Indianapolis, IN, USA) based on the $2^{-\Delta \mathrm{Ct}}$ method (Wang et al. 2016), and all transcript levels were expressed relative to those of the endogenous control TaActin. The whole experiment was repeated twice with independent transgenic lines. Each experiment of 4-12 biological replicates was considered as a block. Calculations of the mean and standard error were performed using Microsoft Excel software. Data were log-transformed to restore normality, and the general linearized model (GLM) ANOVA, Dunnett's test, and two-sample t-test were performed using SAS software version v9.4.

\section{Supplementary Information}

The online version contains supplementary material available at https://doi. org/10.1186/s42483-020-00075-6.

Additional file 1: Table S1. PNPi interactors identified through yeast two-hybrid library screening. Table S2. Raw data for evaluation of wheat resistance to Pst and Pt in TaPR1a-OE transgenic wheat lines. Table S3. Results of inoculation of the transgenic wheat line SP-PNPi-OE with M. oryzae P131. Table S4. Primers used in the current study. Table S5. Statistical analyses for RT-qPCR assays.

Additional file 2: Figure S1. Expression patterns of genes encoding PNPi interactors during barley SAR-like response. Figure S2. Protein interactions between PtPNPi and its possible targets (wNPR1 and TaPR1a) were validated by $\mathrm{Y} 2 \mathrm{H}$ assay. Figure $\mathbf{S 3}$. Evaluation of wheat resistance to stripe rust and leaf rust using ASSESS software. Figure S4. The expression levels of TAPR2 and TaPR4b in transgenic wheat line TaPR1a$O E$ as determined by RT-qPCR assay. Figure S5. Expression patterns of HVAdi3 and HVPR7 during NPR1-mediated acquired resistance. Figure S6. Original, uncropped blots for the Co-IP assay.

\section{Abbreviations}

ABA: Abscisic acid; Ade: Adenine; Adi3: AvrPto-dependent Pto-interacting protein 3; BiFC: Bimolecular fluorescence complementation; Co-IP: Coimmunoprecipitation; DAB: 3,3-diaminobenzidine; DAMP: Damage-associated molecular pattern; dpi: Days post-inoculation; ERF5: Ethylene response factor 5; GFP: Green florescent protein; GLM: General linearized model;

$\mathrm{H}_{2} \mathrm{O}_{2}$ : Hydrogen peroxide; His: Histidine; hpi: Hours post-infiltration; JA: Jasmonate; Leu: Leucine; NBT: Nitroblue tetrazolium; NPR1: Nonexpresser of PR genes $1 ; \mathrm{O}_{2}^{-}$: Superoxide anion; PI-1: Proteinase inhibitor $1 ; \mathrm{PI}-$ 2: Proteinase inhibitor 2; PR: Pathogenesis-related; PRR: Pattern recognition receptor; Pst: Puccinia striiformis f. sp. tritici; Pt: Puccinia triticina; PTI: Patterntriggered immunity; PtPNPi: Puccinia triticina PNPi homolog; RFP: Red florescent protein; ROS: Reactive oxygen species; SA: Salicylic acid; SAR: Systemic acquired resistance; SD: Synthetic dropout; Trp: Tryptophan; WNPR1: Wheat homolog of NPR1 protein; Y2H: Yeast two-hybrid; YFP: Yellow fluorescent protein

\section{Acknowledgments}

We would like to thank Prof. Jorge Dubcovsky (University of California, Davis, United States) for providing the Y2H library, Prof. Genying Li (Shandong Academy of Agricultural Sciences, China) for the excellent support in generating transgenic wheat materials.

\section{Authors' contributions}

XDW and XJW designed the experiment. WSB conducted most of the experiments. SQZ and JJZ performed the RT-qPCR validation. JS worked on the rust inoculation. XDW generated the first and last draft of the manuscript. XMY, DQL, ZSK, XJW and XDW contributed to the revision of the manuscript. All authors read and approved the final manuscript.

\section{Funding}

This work was supported by National Natural Science Foundation of China (31701776), Modern Agricultural Industry Technology System (CARS-03), Provincial Natural Science Foundation of Hebei for Excellent Young Scholar (C2018204091), Technology Innovation Team of Shaanxi Province (2018TD004). Open Project Program of State Key Laboratory of Crop Stress Biology for Arid Areas (CSBAAKF2018009), Provincial Supporting Program of Hebei for the Returned Oversea Scholars (C20190180), and Modern Agricultural Industry System Wheat Innovation Team in Hebei Province

(HBCT2018010204). The funding bodies had no role in design of the study and collection, analysis, and interpretation of data and in writing the manuscript.

\section{Availability of data and materials}

Not applicable.

Ethics approval and consent to participate Not applicable.

\section{Consent for publication}

Not applicable.

\section{Competing interests}

The authors declare that they have no competing interests.

\section{Author details}

'State Key Laboratory of North China Crop Improvement and Regulation, College of Plant Protection, Technological Innovation Center for Biological Control of Crop Diseases and Insect Pests of Hebei Province, Hebei Agricultural University, Baoding, Hebei 071000, People's Republic of China. ${ }^{2}$ College of Life Science, Hebei Agricultural University, Baoding, Hebei 071000, People's Republic of China. ${ }^{3}$ State Key Laboratory of Crop Stress Biology for Arid Areas, College of Plant Protection, Northwest Agriculture and Forestry University, Yangling, Shaanxi 712100, People's Republic of China. 
Received: 1 July 2020 Accepted: 26 October 2020

Published online: 10 November 2020

\section{References}

Backer R, Naidoo S, van den Berg N. The NONEXPRESSOR OF PATHOGENESIS-RELA TED GENES 1 (NPR1) and related family: mechanistic insights in plant disease resistance. Front Plant Sci. 2019;10:102.

Breen S, Williams SJ, Outram M, Kobe B, Solomon PS. Emerging insights into the functions of pathogenesis-related protein 1. Trends Plant Sci. 2017;22:871-9.

Breen S, Williams SJ, Winterberg B, Kobe B, Solomon PS. Wheat PR-1 proteins are targeted by necrotrophic pathogen effector proteins. Plant J. 2016;88:13-25.

Cantu D, Segovia V, Maclean D, Bayles R, Chen X, Kamoun S, et al. Genome analyses of the wheat yellow (stripe) rust pathogen Puccinia striiformis $\mathrm{f}$. sp. tritici reveal polymorphic and haustorial expressed secreted proteins as candidate effectors. BMC Genomics. 2013a;14:270.

Cantu D, Yang B, Ruan R, Li K, Menzo V, Fu D, et al. Comparative analysis of protein-protein interactions in the defense response of rice and wheat. BMC Genomics. 2013b;14:166

Cao H, Bowling SA, Gordon AS, Dong X. Characterization of an Arabidopsis mutant that is nonresponsive to inducers of systemic acquired resistance. Plant Cell. 1994;6:1583-92.

Chen H, Chen J, Li M, Chang M, Xu K, Shang Z, et al. A bacterial type III effector targets the master regulator of salicylic acid signaling, NPR1, to subvert plant immunity. Cell Host Microbe. 2017;22:777-88.

Chen YL, Lee CY, Cheng KT, Chang WH, Huang RN, Nam HG, et al. Quantitative peptidomics study reveals that a wound-induced peptide from PR-1 regulates immune signaling in tomato. Plant Cell. 2014;26:4135-48.

Colebrook EH, Creissen G, Mcgrann GRD, Dreos R, Lamb C, Boyd LA. Broadspectrum acquired resistance in barley induced by the Pseudomonas pathosystem shares transcriptional components with Arabidopsis systemic acquired resistance. Mol Plant-Microbe Interact. 2012;25:658-67.

Curtis MD, Grossniklaus U. A gateway cloning vector set for high-throughput functional analysis of genes in planta. Plant Physiol. 2003;133:462-9.

Delaney TP, Friedrich L, Ryals JA. Arabidopsis signal transduction mutant defective in chemically and biologically induced disease resistance. Proc Natl Acad Sci U S A. 1995;92:6602-6.

Du Y, Mpina MH, Birch PRJ, Bouwmeester K, Govers F. Phytophthora infestans RXLR effector AVR1 interacts with exocyst component Sec5 to manipulate plant immunity. Plant Physiol. 2015;169:1975-90.

Duplessis S, Cuomo CA, Lin YC, Aerts A, Tisserant E, Veneault-Fourrey C, et al. Obligate biotrophy features unraveled by the genomic analysis of rust fungi. Proc Natl Acad Sci U S A. 2011;108:9166-71

Ferreira RB, Monteiro S, Freitas R, Santos CN, Chen Z, Batista LM, et al. The role of plant defence proteins in fungal pathogenesis. Mol Plant Pathol. 2007;8:677-700.

Fu D, Uauy C, Distelfeld A, Blechl A, Epstein L, Chen X, et al. A kinase-START gene confers temperature-dependent resistance to wheat stripe rust. Science. 2009;323:1357-60.

Fu ZQ, Yan S, Saleh A, Wang W, Ruble J, Oka N, et al. NPR3 and NPR4 are receptors for the immune signal salicylic acid in plants. Nature. 2012;486:228-32.

Gamir J, Darwiche R, Van'T Hof P, Choudhary V, Stumpe M, Schneiter R, et al. The sterol-binding activity of PATHOGENESIS-RELATED PROTEIN 1 reveals the mode of action of an antimicrobial protein. Plant J. 2017;89:502-9.

Gao J, Bi W, Li H, Wu J, Yu X, Liu D, et al. WRKY transcription factors associated with NPR1-mediated acquired resistance in barley are potential resources to improve wheat resistance to Puccinia triticina. Front Plant Sci. 2018;9:1486.

Hovmøller MSV, Walter S, Justesen AF. Escalating threat of wheat rusts. Science. 2010;329:369.

Huang S, Zuo S, Zheng D, Liu Y, Du Z, Kang Z, et al. Three formae speciales of Puccinia striiformis were identified as heteroecious rusts based on completion of sexual cycle on Berberis spp under artificial inoculation. Phytopathol Res. 2019;1:14.

Kiran K, Rawal HC, Dubey H, Jaswal R, Devanna BN, Gupta DK, et al. Draft genome of the wheat rust pathogen (Puccinia triticina) unravels genome-wide structura variations during evolution. Genome Biol Evol. 2016;8:2702-21.

Lamari L. Assess 2.0 : image analysis software for plant disease quantification. St Paul: The American Phytopathological Society (APS); 2008.

Li H, Wu J, Shang X, Geng M, Gao J, Zhao S, et al. WRKY transcription factors shared by BTH-induced resistance and NPR1-mediated acquired resistance improve broad-spectrum disease resistance in wheat. Mol Plant-Microbe Interact. 2020;33:433-43.
Li Q, Chen Y, Wang J, Zou F, Jia Y, Shen D, et al. A Phytophthora capsici virulence effector associates with NPR1 and suppresses plant immune responses. Phytopathol Res. 2019;1:6.

Liu C, Pedersen C, Schultz-Larsen T, Aguilar GB, Madriz-Ordeñana K, Hovmøller MS, et al. The stripe rust fungal effector PEC6 suppresses pattern-triggered immunity in a host species-independent manner and interacts with adenosine kinases. New Phytol. 2016;213:1556.

Lu S, Faris JD, Sherwood R, Friesen TL, Edwards MC. A dimeric PR-1-type pathogenesis-related protein interacts with ToxA and potentially mediates ToxA-induced necrosis in sensitive wheat. Mol Plant Pathol. 2014;15:650-63.

Ma L, Djavaheri M, Wang H, Larkan NJ, Haddadi P, Beynon E, et al. Leptosphaeria maculans effector protein AvrLm1 modulates plant immunity by enhancing MAP kinase 9 phosphorylation. iScience. 2018;3:177-91.

Molina A, Görlach J, Volrath S, Ryals J. Wheat genes encoding two types of PR-1 proteins are pathogen inducible, but do not respond to activators of systemic acquired resistance. Mol Plant-Microbe Interact. 1999;12:53-8.

Mou Z, Fan W, Dong X. Inducers of plant systemic acquired resistance regulate NPR1 function through redox changes. Cell. 2003;113:935-44.

Paolacci AR, Tanzarella OA, Porceddu E, Ciaffi M. Identification and validation of reference genes for quantitative RT-PCR normalization in wheat. BMC Mol Biol. 2009;10:11.

Petre B, Saunders DG, Sklenar J, Lorrain C, Krasileva KV, Win J, et al. Heterologous expression screens in Nicotiana benthamiana identify a candidate effector of the wheat yellow rust pathogen that associates with processing bodies. PLoS One. 2016;11:e0149035.

Ryals J, Lawton KA, Delaney TP, Friedrich L, Kessmann H, Neuenschwander U, et al. Signal transduction in systemic acquired resistance. Proc Natl Acad Sci U S A. 1995;92:4202-5.

Ryals J, Weymann K, Lawton K, Friedrich L, Ellis D, Steiner HY, et al. The Arabidopsis NIM1 protein shows homology to the mammalian transcription factor inhibitor I kappa B. Plant Cell. 1997;9:425-39.

Saleh A, Withers J, Mohan R, Marqués J, Gu Y, Yan S, et al. Posttranslational modifications of the master transcriptional regulator NPR1 enable dynamic but tight control of plant immune responses. Cell Host Microbe. 2015;18: 169-82.

Sels J, Mathys J, De Coninck BMA, Cammue BPA, De Bolle MFC. Plant pathogenesis-related (PR) proteins: a focus on PR peptides. Plant Physiol Biochem. 2008:46:941-50.

Shah J, Tsui F, Klessig DF. Characterization of a salicylic acid-insensitive mutant (sai1) of Arabidopsis thaliana, identified in a selective screen utilizing the SAinducible expression of the tms2 gene. Mol Plant-Microbe Interact. 1997;10: 69-78.

Silva KJP, Mahna N, Mou Z, Folta KM. NPR1 as a transgenic crop protection strategy in horticultural species. Hortic Res. 2018;5:15.

Smith SM, Li C, Li J. Hormone function in plants. In: Li J, Li C, Smith SM, editors. Hormone metabolism and signaling in plants. London: Academic Press; 2017 p. 1-38.

van Loon LC, Rep M, Pieterse CMJ. Significance of inducible defense-related proteins in infected plants. Annu Rev Phytopathol. 2006;44:135-62.

Wang F, Yuan S, Wu W, Yang Y, Cui Z, Wang H, et al. TaTLP1 interacts with TaPR1 to contribute to wheat defense responses to leaf rust fungus. PLoS Genet. 2020;16:e1008713.

Wang X, Bi W, Gao J, Yu X, Wang H, Liu D. Systemic acquired resistance, NPR1, and pathogenesis-related genes in wheat and barley. J Integr Agric. 2018;17: 2468-77.

Wang X, Yang B, Li K, Kang Z, Cantu D, Dubcovsky J. A conserved Puccinia striiformis protein interacts with wheat NPR1 and reduces induction of pathogenesis-related genes in response to pathogens. Mol Plant-Microbe Interact. 2016:29:977-89.

Wawra S, Trusch F, Matena A, Apostolakis K, Linne U, Zhukov I, et al. The RxLR motif of the host targeting effector AVR3a of Phytophthora infestans is cleaved before secretion. Plant Cell. 2017:29:1184-95.

Withers J, Dong X. Posttranslational modifications of NPR1: a single protein playing multiple roles in plant immunity and physiology. PLOS Pathog. 2016; 12:e1005707

Wu J, Gao J, Bi W, Zhao J, Yu X, Li Z, et al. Genome-wide expression profiling of genes associated with the $L r 47$-mediated wheat resistance to leaf rust (Puccinia triticina). Int J Mol Sci. 2019;20:4498.

Yang B, Ruan R, Cantu D, Wang X, Ji W, Ronald PC, et al. A comparative approach expands the protein-protein interaction node of the immune receptor XA21 in wheat and rice. Genome. 2013;56:315-26. 
Yang G, Tang L, Gong Y, Xie J, Fu Y, Jiang D, et al. A cerato-platanin protein SsCP1 targets plant PR1 and contributes to virulence of Sclerotinia sclerotiorum. New Phytol. 2017;217:739-55.

Zhang W, Chen S, Abatea Z, Nirmala J, Rouse MN, Dubcovsky J. Identification and characterization of Sr13, a tetraploid wheat gene that confers resistance to the Ug99 stem rust race group. Proc Natl Acad Sci U S A. 2017;114:E9483-92.

Zhao S, Shang X, Bi W, Yu X, Liu D, Kang Z, et al. Genome-wide identification of effector candidates with conserved motifs from the wheat leaf rust fungus Puccinia triticina. Front Microbiol. 2020;11:1188.

Zheng W, Huang L, Huang J, Wang X, Chen X, Zhao J, et al. High genome heterozygosity and endemic genetic recombination in the wheat stripe rust fungus. Nat Commun. 2013;4:2673.

Ready to submit your research? Choose BMC and benefit from:

- fast, convenient online submission

- thorough peer review by experienced researchers in your field

- rapid publication on acceptance

- support for research data, including large and complex data types

- gold Open Access which fosters wider collaboration and increased citations

- maximum visibility for your research: over $100 \mathrm{M}$ website views per year

At BMC, research is always in progress.

Learn more biomedcentral.com/submissions 